\title{
Automated Placement Coordinator System with Automated Proctoring Assessment using Python Django
}

\author{
J. Antony Jerold ${ }^{1}$, A. George Antony ${ }^{1}$, S. Hariharan ${ }^{1}$, \\ L. Saranhariharajeyan ${ }^{1}$, Prof P. Sowkarthika ${ }^{2}$, Dr. N. Suguna ${ }^{3}$ \\ ${ }^{1}$ UG Scholar, Department of Computer Science and Engineering, Akshaya College of Engineering and Technology, \\ Coimbatore, Tamil Nadu, India \\ ${ }^{2}$ Assistant Professor, Department of Computer Science and Engineering, Akshaya College of Engineering and \\ Technology, Coimbatore, Tamil Nadu, India \\ ${ }^{3}$ Professor, Department of Computer Science and Engineering, Akshaya College of Engineering and Technology, \\ Coimbatore, Tamil Nadu, India
}

\begin{abstract}
The Automation software plays several tasks in day-to-day life. Lightning speed of this technology growth in various domain reduces time and gives trusted benefits. For instance, consider a typical student's placement has to face many challenges to be hired. The placement of the students based on their own academics and technical skills that is required by recruiter. Considering that, the educational institutes devise students to achieve their placement goals. For instance, the institute has to arrange placement training session to students for regular practice. The regular practice lets student to mind tedious it has to be transpose by making a competition among the students. This can be achieved by making up the practice and problem solving tasks by online test and giving the credit scores based upon their performance on tasks. By considering their credit scores the daily, weekly, academic leaderboard can be prepared among the students which make interest on learning. This tasks may be bypassed by students during test so we make this in a secure way by Automated Proctoring. Considering all this commodity student report can be easily generated by system based on recruiter requirements that reduces the time of recruitment and also student can come up with the magnificent skillset for Placement.
\end{abstract}

Keywords: Automated Proctoring, Online Assessments, Automated Placement Prediction Algorithm, Event Managements, Students Leaderboard.

\section{INTRODUCTION}

System main aim is to provide a beneficial knowledge by online training and assessments to students for their placement and to produce trusted report based on students' performance which understand the recruiters obligation. Automated Placement Coordinating system is a digital platform that would enable individual profile to students offers easy leaning platform that helps in placement. It has its own online training tasks and Automated proctoring assessments with credit scores which helps in producing daily, weekly, academic leaderboard among students and also a trusted report based on recruiter requirements. The academic performance of individual student is maintained with our system regularly. The assessments include aptitude, problem solving, Technical Programming which includes C, C++, Java, Python, C\#, etc. Automated Proctoring captures image, audio, motion, and systemic changes to identify any abnormal assessments-taking behaviors. However, once the proctoring session is complete, Automated Proctoring provides an trust scale report which affect the credit score of the assessments. Which helps to prevent student from bypassing the assessments. APPA is the algorithm Figure: 1.2 created for the Automated Placement Coordinating system which helps to predict the student performance and skills with recruiter helps to understand the working model of APPA.

\section{LITERATURE SURVEY}

Paper 1: This system works in two different modules namely, MCQ and Handwriting Analysis. The MCQ module consists of aptitude questions and psychometric questions. The aptitude test gives the top five appropriate broad career domains. The psychometric test gives an idea about conscious personality traits and a few specific careers. The handwriting analysis gives specific careers. 


\section{International Advanced Research Journal in Science, Engineering and Technology}

Vol. 8, Issue 5, May 2021

\section{DOI: $10.17148 /$ IARJSET.2021.8580}

Paper 2: System uses 5 aspects related to aptitudes in this research such as verbal analysis test, visual reasoning test, numerical reasoning test, spatial recognition test and vocabulary test. The Rasch model was used to analyze the difficulty level of the item, and from the results of the study showed that the difficulty level of the item was in the normal distribution at the interval -4.5735 to 6.4989 .

Paper 3: With the help of augmented reality technology that combines the real world with a virtual world, be it a two dimensional and Machine learning is an application of artificial intelligence that provides systems the ability to automatically learn and improve from experience. This interview training simulator which helps users to built on their technical skills and also create a interview experience to the user virtually.

Paper 4: The present research investigates whether applicants engage in dishonest behaviours across selection hurdles. It place two opposite conceptual views on trial: (a) the behavioural consistency view predicting that applicants should display consistent behaviour patterns (honest or dishonest) across selection hurdles, and (b) the situational specificity view positing that dishonest behaviours do not necessarily generalize across selection hurdles.

Paper 5: The automatic exam activities recognition is necessitating and a demanding field of research. In this research work, categorization of students' activities during the exam is performed using a deep learning approach. A new deep Convolutional Neural Network $(\mathrm{CNN})$ architecture with 46 layers is proposed which contains the characteristics of deep AlexNet and SqueezeNet.

Paper 6: This study collected the evaluation and feedback of college students from different universities and different majors on online teaching mode and teaching platform, conducted a quantitative study of SPSS samples, and analysed the influence of learners' and teachers' participation on online teaching effect.

Paper 7: This study includes the cost of the service and their technical requirements that help to evaluate the online proctoring tools. This paper reports on one of the first attempts to properly evaluate a selection of these tools and offer recommendations for educational institutions.

Paper 8: Twenty-seven Chilean EFL teacher candidates participated in this interpretative case study aiming at exploring the challenges and opportunities of this virtual teaching experience. The results indicate that factors such as the lack of direct interaction with learners and the sudden change of setting were among those that most strongly affected the participants own learning process.

\section{PROPOSED SYSTEM}

The proposed system aims at creating a digitalized platform to present a trusted report of student based on his academic and technical performance which is required for recruiters requirement. Automated placement coordinating system helps to arrange assessment like aptitude and technical programming with $\mathrm{C}, \mathrm{C}++$, Java, Python, etc for the students in a secure way by automated proctoring.

Automated Proctoring which enable us a secured way of conducting assessments. It uses the device camera and microphone. Camera used to record the live video of student during assessments and uses face recognition technique for unusual activities. Microphone is used to record the background sounds which also helps to prevent unusual activities. Proctoring also track the system application which prevent from using other application other than assessment application during the time of assessment.

Automated proctoring can be performed by face recognition which uses the concept of cosine similarity. The system can infer baye's rule to allocate student trust report during assessment.

\section{Advantages}

A secured trusted student report by APPA which is required for recruiter can be generated easily.

$\checkmark \quad$ Regular practice of tasks by automated placement coordinating system helps to crack any level of problem solving questions.

$\checkmark \quad$ It introduces new generation of leaning environment among the students.

$\checkmark \quad$ Fake assessment result is completely reduced with the help of automated proctoring

$\checkmark \quad$ Reduces the time of candidate selection process.

\subsection{Process of Student Selection}

For Generating a trusted report of individual students the following things has to be done regularly,

$\checkmark \quad$ Academic Scores are updated regularly.

$\checkmark \quad$ New task is created regularly.

$\checkmark \quad$ The student can access regular task in order to develop their skills

$\checkmark \quad$ Credit score for the task is generated by performance of the assessments and automated proctoring trust report.

Automated placement prediction algorithm analysis the student scores in both academic and technical skills which is store by conducted through automated proctoring in secured way. If the recruiter selects his/her requirement for organization which involves education scores and technical knowledge of students. APPA generate a trusted student report for the recruiter obligation. 


\title{
3.2. Score Calculation
}

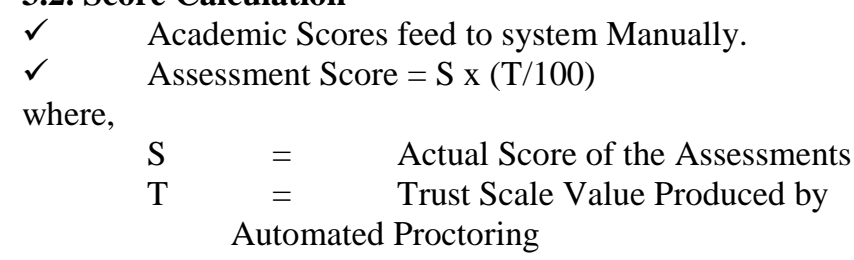

\subsection{Steps To Create New Assessment}

\author{
$\checkmark \quad$ Select Add Assessment in Admin panel \\ $\checkmark \quad$ Name the assessment \\ $\checkmark \quad$ Create assessment With multiple choice question. \\ $\checkmark \quad$ Complete creating by create option.
}

\subsection{Steps To Create Appa Student Report}

$\checkmark \quad$ Select APPA Report in Admin panel

$\checkmark \quad$ Fill out the form based on the recruiter requirements.

$\checkmark \quad$ Select generate which display report of students based on the students performance which can be exported for future use.

\subsection{Steps To Access Assessments}

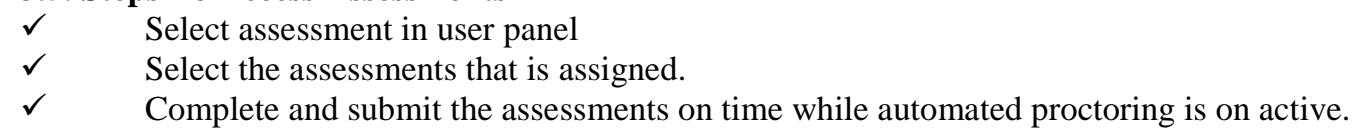

\subsection{Modules}

\subsubsection{Home Page}

The Home page consists of,

$\checkmark \quad$ User login.

$\checkmark \quad$ New user registration.

\subsubsection{User Login}

The user login panel consist of user authentication form which lets the user to login into Automated placement coordinating system.

\subsubsection{New User Registration}

The new user registration form allows to create a new user for Automated placement coordinating system.

\subsubsection{Admin Page}

The Admin page consist of,

$\checkmark \quad$ Dashboard.

$\checkmark \quad$ Event Creation Page.

$\checkmark \quad$ Assessment Creation Section.

$\checkmark \quad$ APPA Report Generation Page.

$\checkmark \quad$ User Access Control Page.

\subsubsection{Dashboard}

Admin Dashboard page shows the live feed of student leaderboard, and displays the short hint on total assessment, No.of.Events along with calendar.

\subsubsection{Event Creation Page}

Event Creation page present only in admin page which helps admin to create a instance to students about organizations Events.

\subsubsection{Assessment Creation Section}

In Assessment Creation Section admin can create Topic, Assessment Name \& Assessment for Students.

\subsubsection{Appa Report Generation Page}

APPA report generation page allows admin to select the recruiter requirements and a report can be generated as per the requirements in a PDF format. 


\section{International Advanced Research Journal in Science, Engineering and Technology}

Vol. 8, Issue 5, May 2021

\section{DOI: 10.17148/IARJSET.2021.8580}

\subsubsection{User Access Control Page}

User Access Control Page only allow admin to manage the users of entire system by password recovery, User Control, etc.

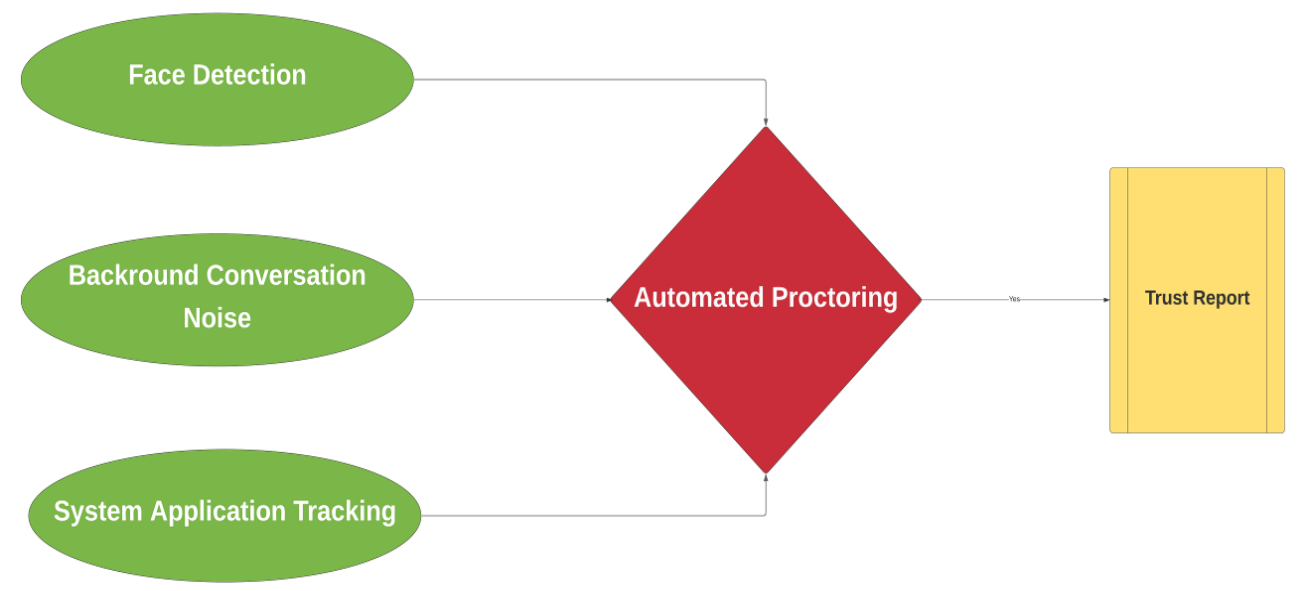

Figure 1. Workflow of Automated Proctoring

\subsubsection{User Page}

The User page consist of,

$\checkmark \quad$ Dashboard

$\checkmark \quad$ Assessment Section

$\checkmark \quad$ Events Page

\subsubsection{Dashboard}

User Dashboard page shows the live feed of student leaderboard, and displays the short hint on pending assessment, Completed assessments, Success rate, Events along with calendar.

\subsubsection{Assessment Section}

Assessment Section allows user to take Automated proctored assessment and allows user to see the history of assessment result.

\subsubsection{Events Page}

Events page enables user to track the upcoming and live events of particular organization.

\section{SYSTEM IMPLEMENTATION}

Following figures describes about the implementation and workflow of Entire System.

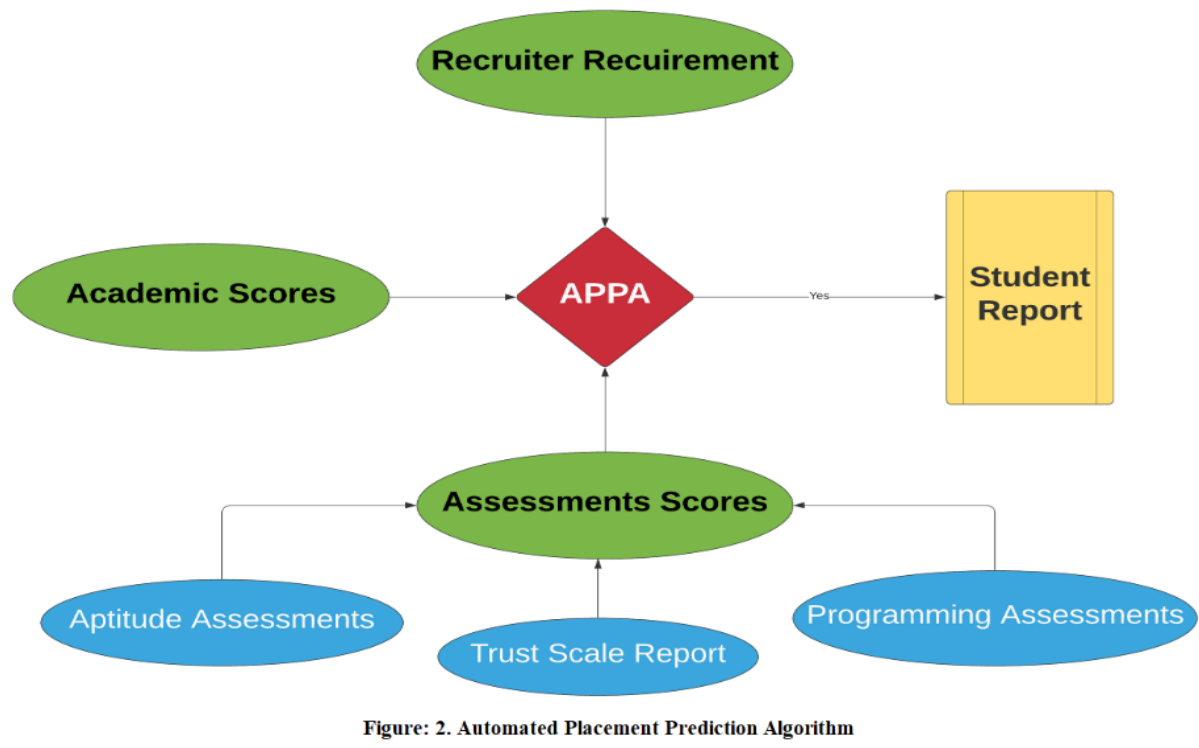




\section{International Advanced Research Journal in Science, Engineering and Technology}

Vol. 8, Issue 5, May 2021

DOI: $10.17148 /$ IARJSET.2021.8580

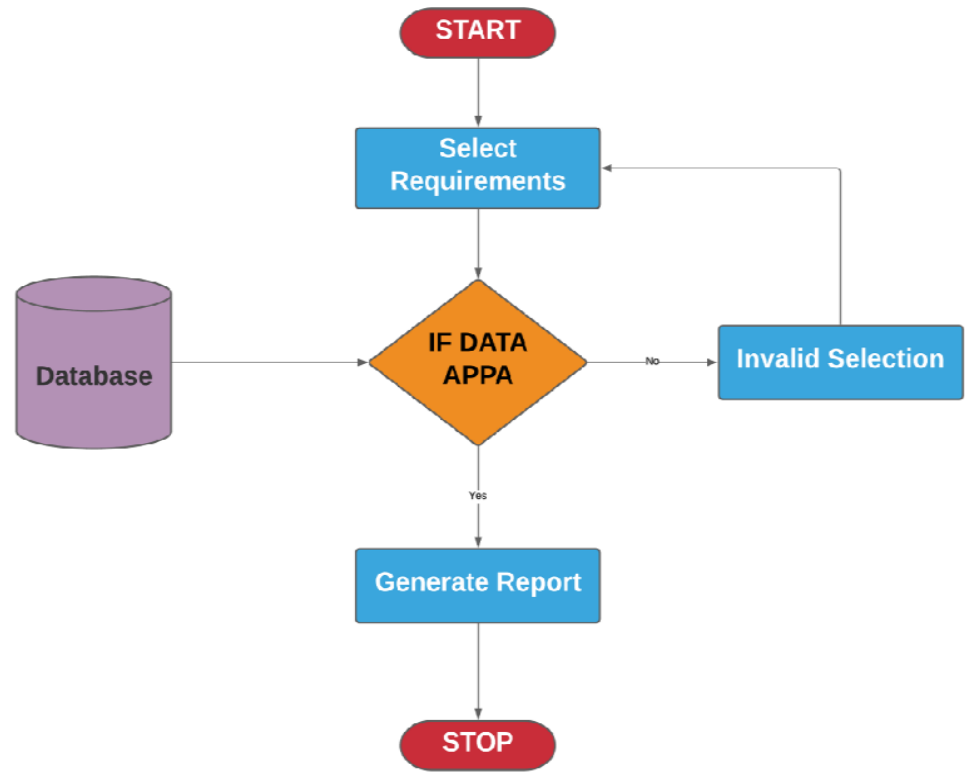

Figure: 3. Workflow of Assessment

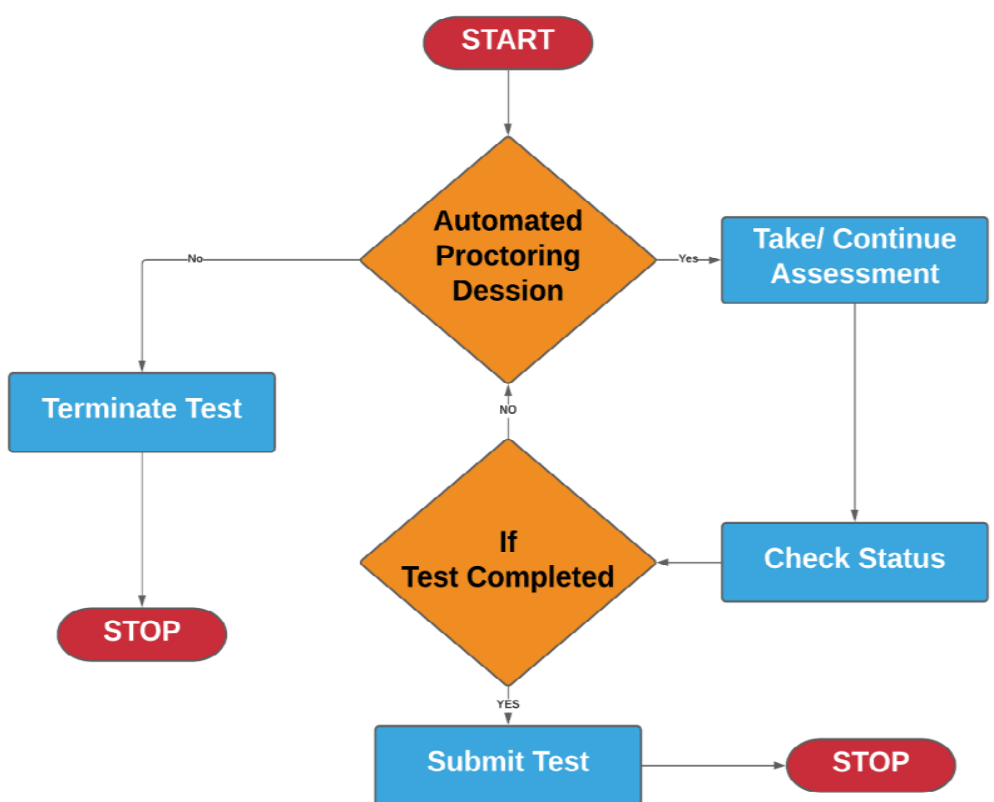

Figure: 4. Workflow of APPA

\section{CONCLUSION}

Since Automated Placement coordinating System has gain a greater attraction in the field of education, the system proposes an approach new leaning method among students, produces trusted report of individual student and reduces the time of placement coordinator as well as recruiter. System is designed to overcome the issues in existing similar systems. Core part of the system was achieved using two methodologies that are Automated proctoring assessments and APPA Algorithm selection process. The automated proctoring use the device camera microphone to record video and audio and also monitor device screen whether the candidate switch between other applications during assessment which helps to understand the student behavior and APPA predicts the students skills and recruiters needs and generate a trusted report and create a leaderboard among students.

\section{Future Scope}

In the Proposed system, All the assessments are carried only through multiple choice questions. In future programming assessments can be performed in online interpreter along with test case for better performance and increases security. System's training carried only through notes and study materials it can also be bring to next level by making live video 


\section{International Advanced Research Journal in Science, Engineering and Technology}

Vol. 8, Issue 5, May 2021

\section{DOI: $10.17148 / I A R J S E T .2021 .8580$}

training session to students. Various additional modules like student forum for discussion between student about any quarries, live chat support can be upgrade to the current System.

\section{REFERENCES}

[1]. Apoorva Anand, Mrs. Dhanashri Patil, Shivani Bhagwat, Suyog Karanje, Vihang Mangalvedhekar, "Automated Career Guidance using Graphology,Aptitude Test and Personality Test", Institute of Electrical and Electronics Engineers (IEEE 2018).

[2]. M. Khairudin, F. Wulandari, D Mardapi, "Implementation of Computer Bases Assessment on Students" Aptitude Using Online and Multimedia Test for Talent Mapping', 5th International Conference on Technology and Vocational Teachers (ICTVT 2019).

[3]. Prajwal Ashok Nagrale, Hrishikesh Ratnaparkhi, Niteen Jogdand, Mohit Shailesh Kulkarni, "Interview Training Simulator", Mukt Shabd Journal (2020).

[4]. Ying Liua, Ji ayi Liub, Guoxiang Zhaoc, Jinyan Fand, "Cheaters on an aptitude test in an earlier selection hurdle are not more likely than noncheaters to fake on a personality test in a subsequent selection hurdle", Personality and Individual Differences (2020).

[5]. Tanzila Saba, Nor Shahida Mohd Jamail, Mudassar Raza, Muhammad Sharif, "Categorizing the Students Activities for Automated Exam Proctoring Using Proposed Deep L2-GraftNet CNN Network and ASO Based Feature Selection Approach", Institute of Electrical and Electronics Engineers (IEEE 2021).

[6]. Jiangxue Zhang, Kaiquan Chen, "Analysis of the Cores of the Improvement of Online Teaching System and Model Based on the Evaluation and Feedback on the Online Teaching Model and Teaching Platform", Journal of Educational Technology Development and Exchange (JETDE 2020).

[7]. Mohammed Juned Hussein, Javed Yusuf, Arpana Sandhya Deb, Letila Fong, Som Naidu, "An Evaluation of Online Proctoring Tools", International Council for Open and Distance Education (ICDE 2020)

[8]. Paulina Sepulveda-Escobar, Astrid Morrison, "Online teaching placement during the COVID-19 pandemic in Chile: challenges and opportunities", European Journal of Teacher Education (EJTE 2020). 